\title{
Marco Nuti, Metamorfosi del sogno: fantasmagorie e deliri onirici in Maupassant, Proust, Baudelaire, Breton e Michaux
}

\section{Mariangela Napolitano}

\section{(2) OpenEdition \\ 1 Journals}

\section{Edizione digitale}

URL: http://journals.openedition.org/studifrancesi/6486

DOI: $10.4000 /$ studifrancesi.6486

ISSN: 2421-5856

\section{Editore}

Rosenberg \& Sellier

\section{Edizione cartacea}

Data di pubblicazione: 1 novembre 2010

Paginazione: 607-608

ISSN: 0039-2944

\section{Notizia bibliografica digitale}

Mariangela Napolitano, «Marco Nuti, Metamorfosi del sogno: fantasmagorie e deliri onirici in Maupassant, Proust, Baudelaire, Breton e Michaux», Studi Francesi [Online], 162 (LIV | III) | 2010, online dal 30

novembre 2015, consultato il 11 janvier 2021. URL: http://journals.openedition.org/studifrancesi/6486 ; DOI: https://doi.org/10.4000/studifrancesi.6486

Questo documento è stato generato automaticamente il 11 janvier 2021.

\section{cc) (†) $\odot$}

Studi Francesi è distribuita con Licenza Creative Commons Attribuzione - Non commerciale - Non opere derivate 4.0 Internazionale. 


\title{
Marco Nuti, Metamorfosi del sogno: fantasmagorie e deliri onirici in Maupassant, Proust, Baudelaire, Breton e Michaux
}

\author{
Mariangela Napolitano
}

\section{NOTIZIA}

MARCO NUTI, Metamorfosi del sogno: fantasmagorie e deliri onirici in Maupassant, Proust, Baudelaire, Breton e Michaux, Fasano, Schena Editore, 2009, pp. 120.

«Straniero, sono inspiegabili e ambigui i sogni, e non tutto si attua per gli uomini». Con un'efficace citazione omerica Marco NUTI apre la parte introduttiva del suo interessante studio sulla metamorfosi del sogno in cinque tra i più importanti scrittori francesi che del sogno hanno intriso le rispettive opere: Maupassant, Proust, Baudelaire, Breton e Michaux. Il sogno come evento mentale, divino o profetico che sia è sempre stato richiamo della letteratura, in particolar modo dal periodo romantico ai nostri giorni. Forse perché finché ci è dato sognare, in qualche modo ci è permesso di raccontarlo o semplicemente perché è solo come esposizione narrativa che il sogno smette di essere un flusso onirico sfuggente e acquisisce sostanza. Sostanza che diventa materia prima di una sorta di ponte tra una dimensione strettamente sensoriale e quella rappresentazionale della realtà. Ed è proprio questo ponte che diventa fulcro dello studio dell'autore, in quanto luogo in cui tutto sembra azzerarsi permettendo così la nascita di nuovi codici letterari.

2 Il volume è organizzato in cinque parti ognuna rispondente alle diverse esperienze oniriche e letterarie dei vari scrittori. La prima, «Poetiche del sogno nei racconti di Maupassant» (pp. 13-30), prendendo in considerazione in particolar modo il racconto fantastico Le Horla (1887), riflette l'approccio al sogno dello scrittore normanno, non 
più romantico, ma scientifico, quasi a voler demistificare l'aspetto soprannaturale che da sempre era stato conferito all'onirico. L'analisi fa riferimento alla seconda versione della nouvelle fantastique, non solo più analitica e funzionale, ma anche più nota della sua precedente. Maupassant, o quanto meno i suoi personaggi, sembrano in qualche modo voler abbandonare il campo del meraviglioso quale lo definisce Todorov, per approdare al campo del fantascientifico dove le stesse leggi e meccanismi della scienza fungono da veicolo per condurre alla soluzione di ogni evento "fantastico". Scienza che però resta quella ambigua e decadente di fine Ottocento che più che risolvere le angosce umane, apre varchi sull'ignoto per poterne avere di nuove. Ciò che all'autore preme sottolineare è come il sogno diventi fonte di una nuova creatività che si ritrova ad essere, allo stesso tempo, nuovo spazio e nuovo limite. Maupassant, allora, non diventa altro che scrittore di questa nuova dicotomia, di questo "ponte" che segna l'alterità dell'ignoto e al tempo stesso ci avvicina ad esso.

3 La seconda parte, intitolata «Cattedrali gotiche nell'eternità: Proust e l'imagerie» (pp. 31-47), si avvale di un racconto onirico presente ne Le côté de Guermantes I, in cui il narratore della Recherche racconta il suo «inatteso e nebuloso torpore». Anche quello di Proust per il sogno può essere considerato un interesse di tipo scientifico piuttosto che una semplice fascinazione. Il padre era un medico e, grazie ai suoi libri, Marcel si era ampiamente documentato circa la neurofisiologia del sogno. Come in Maupassant, quindi, nulla di "romantico" nel suo approccio. Marco Nuti si sofferma sulla particolarità dell'universo onirico proustiano che è prettamente liquido: i sogni sono spesso legati a immagini marine perché il sonno, così come l'acqua, sembra cancellare i ricordi sottraendo il sognatore ai vincoli della contingenza. È l'acqua quindi in questo caso a fare da ponte, al tempo stesso rigenerandolo e richiamandolo verso l'infinito del sogno. Fondamentale è dunque l'attività onirica nell'universo letterario di Proust, in quanto aiuta il lavoro del ricordo e quindi della memoria, unica custode della verità.

«Ode all'Odalisca: la vertebra del sogno ne La chambre double di Charles Baudelaire» (pp. 49-69), terza parte dello studio, fa leva sulla distinzione, che intercorre all'interno di Les paradis artificiels, tra il rêve naturel e il rêve absurde. Il primo, legato alla vita diurna, alla quotidianità, è espressione naturale dell'uomo e fa capo alla sua memoria volontaria; il secondo, legato al misterioso, al soprannaturale, al non-visibile, fa capo invece alla memoria involontaria dell'uomo. Una differenza che si rispecchia e si bilancia in quella più vasta e immediata che contrappone il sogno alla realtà e che permette a Baudelaire, così come allo stesso Nuti, di sottolineare l'essere doppio del sogno. Esempio e strumento per poter attraversare lo scenario spesso "anti-naturalistico" del paesaggio onirico è la pittura e in particolar modo quella di due artisti molto amati da Baudelaire: Delacroix e Ingres. Nuti si era già occupato dello stretto legame che unisce le due forme d'Arte, come è possibile notare nel suo Et in pictura fabulator: Paul Cézanne et le dialogue créateur entre peinture, littérature et philosophie de Balzac à Maldiney (2008). Qui evidenzia come il linguaggio poetico, utilizzato per raccontare il sogno, assimili la poesia alla descrizione di un quadro. Così come Ingres dà movimento alla sua Odalisca attraverso la sua invisibile vertebra in più, così il poeta dà a vedere la sua poesia, conferendole il peso di una nuova visione e la leggerezza di una nuova libertà formale.

5 Al poeta maudit segue il padre del surrealismo, nella quarta parte del volume intitolata «Dis-moi qui te hante, je te dirai qui tu es: Breton e i fantasmi di Nadja» (pp. 71-83). Se i Romantici ne avevano fatto un dualismo, i Surrealisti tentano ora di riunire ciò che prima di loro era stato spezzato. Così il sogno e la realtà si incontrano per creare una 
sorta di terzo spazio, una Realtà superiore, in cui rifuggire il mondo borghese esplorando il vuoto dell'inconscio. Solo l'immaginazione rende visibile il fantasma di Nadja, e tutto quanto di spettrale e disincarnato l'opera di Breton contenga. La libertà dello spirito umano smette di fondarsi sulla precarietà della vita reale quotidiana $\mathrm{e}$ comincia a fare affidamento non più alla realtà, ma alla possibilità di essa, attraverso l'unico mezzo e strumento possibile: l'immaginazione. "Osare l'inimmaginabile», insomma.

Quinta ed ultima parte è «Scritture della fenice: Michaux e il sogno drogato» (pp. 85-103). Parlare di onirico in Michaux significa parlare di pratiche esoteriche che creano una continuità tra reale e immaginario un po' diversa da quanto analizzato finora, con descrizioni continue di sonno e veglia che non possono che far deragliare chi si accinge alla lettura. Michaux approda ad un sogno appesantito dal forte potere onirogeno della mescalina che crea un limite sottilissimo tra l'allucinazione per droga e l'allucinazione per sogno. Il "ponte" fornito dalle sostanze allucinogene, è un ponte barcollante che elimina lo scarto tra l'universo esterno e il mondo interiore. Il racconto di ciò che avviene o si crede sia avvenuto è una testimonianza retrospettiva $\mathrm{e}$ incompleta, così come l'allucinazione stessa è un tempo passato e bucato perché non fissato dalla memoria. Nuti osserva quindi come il linguaggio non sia in grado, in questo caso, di tradurre tali visioni, di colmare le lacune, riempire i buchi. Per questo Michaux crea continuamente neologismi, nuove costruzioni lessicali, sostituendo spesso le parole con disegni. La sua scrittura, spesso quasi cinematografica, è una devozione alla visione e ciò rende la sua poesia penetrante come mai nessuna prima. 